\title{
Pause by ECG Finding
}

National Cancer Institute

\section{Source}

National Cancer Institute. Pause by ECG Finding. NCI Thesaurus. Code C119250.

An electrocardiographic recording that demonstrates an R-R interval that exceeds a predefined duration threshold, irrespective of the underlying rhythm. 